\title{
NLTE abundances of Mn in a sample of metal-poor stars ${ }^{\star}$
}

\author{
M. Bergemann ${ }^{\star}$ and T. Gehren
}

\author{
Institute for Astronomy and Astrophysics, Ludwig-Maximilian University, Scheinerstr. 1, 81679 Munich, Germany \\ e-mail: masha@rejoin.net
}

Received 30 April 2008 / Accepted 3 October 2008

ABSTRACT

\begin{abstract}
Aims. Following our solar work, we perform NLTE calculations of the Mn abundance for fourteen stars with [Fe/H] from 0 to -2.5 , mainly to show how NLTE affects Mn abundances in cool stars of different metallicities.

Methods. The spectrum synthesis and Mn abundances are based on statistical equilibrium calculations using various estimates for the influence of hydrogen collisions.

Results. The NLTE abundances of Mn in all studied stars are systematically higher than the LTE abundances. At low metallicities, the NLTE abundance corrections may run up to $0.5-0.7$ dex. Instead of a strong depletion of Mn relative to Fe in metal-poor stars as found by the other authors, we only find slightly subsolar values of $[\mathrm{Mn} / \mathrm{Fe}]$ throughout the range of metallicities analyzed here. Conclusions. The $[\mathrm{Mn} / \mathrm{Fe}]$ trend in metal-poor stars is inconsistent with the predictions of galactic chemical evolution models, where $\mathrm{Mn}$ is less produced than $\mathrm{Fe}$.
\end{abstract}

Key words. line: profiles - line: formation - stars: abundances - nuclear reactions, nucleosynthesis, abundances

\section{Introduction}

The abundances of manganese in stars of different populations have been extensively studied during the past decades (Gratton 1989; Ryan et al. 1996; Nissen et al. 2000; Prochaska \& McWilliam 2000; see also the review of Wheeler et al. 1989). Although different methods and assumptions were applied to determine the Mn abundances, they all agree that this element is deficient compared to iron in metal-poor stars. The degree of the derived $\mathrm{Mn}$ depletion is claimed to vary from the thin disk to the halo and bulge populations, and from cluster to field stars (Gratton 1989), and it is different for the Milky Way and the Sgr dwarf galaxy (Prochaska \& McWilliam 2000).

Different assumptions are invoked to explain the trends, but they cannot reconcile all observational facts simultaneously, especially when other elements of the iron group $(\mathrm{Cr}, \mathrm{Co}$, and $\mathrm{Ni}$ ) are considered. A critical issue is the main astrophysical production site of manganese. While some favor the hypothesis of a Mn overproduction with respect to $\mathrm{Fe}$ in supernovae of type Ia (Prochaska \& McWilliam 2000; Nissen et al. 2000; Sobeck et al. 2006); McWilliam et al. (2003) suggest that metallicitydependent yields from type II supernovae can explain the observed abundance trend. Both hypotheses imply that the $\mathrm{Mn} / \mathrm{Fe}$ ratio in supernovae grows with decreasing metal abundance.

No reliable constraint to Mn nucleosynthesis can be drawn from models of late stellar evolution, because stellar yields depend on many factors that are poorly known. As was shown among others by Umeda \& Nomoto (2002), the position of the model mass cut influences the ratio of abundances originating from the alpha-rich freeze-out and from the incomplete $\mathrm{Si}$ burning. This affects the abundances of $\mathrm{Mn}, \mathrm{Cr}$, and Co produced by the decay of ${ }^{55} \mathrm{Co},{ }^{52} \mathrm{Fe}$, and ${ }^{59} \mathrm{Cu}$, respectively. The explosion

\footnotetext{
* Based on observations collected at the European Southern Observatory, Chile, 67.D-0086A, and the Calar Alto Observatory, Spain.

$\star \star$ Research supported by the International Max Planck Research School (IMPRS), Munich, Germany.
}

energy is another free parameter in SN nucleosynthesis calculations (Heger \& Woosley 2008), which changes the yields of a number of iron group elements simultaneously. Because of this, the interpretation of yields becomes very non-trivial.

As noted above, different methods are used to calculate the Mn abundances. One detail, however, is common to all analyses so far. The assumption of LTE, which was shown to depart from reality for many elements, in particular for metal-poor stars. Most of the NLTE investigations refer to the lighter elements, such as C, N, O (Asplund et al. 2005a,b; Takeda \& Honda 2005) or $\mathrm{Na}, \mathrm{Mg}$, and $\mathrm{Al}$ (Gehren et al. 2006), but some also deal with heavier elements, among them Mashonkina et al. (2007: Ca), Korn et al. (2003: Fe), Bruls (1993: Ni), Mashonkina et al. (2003, 2008: Sr, Ba, and Eu). The purpose of this paper is to show the influence of NLTE effects on the determination of Mn abundances for a sample of reference stars. At present, we do not pretend to revise the observed trend completely, but even from our small sample we conclude that the difference between LTE and NLTE Mn abundances may become large in metalpoor stars. The use of LTE Mn abundances in modeling Galactic chemical evolution is therefore not supported.

The paper is set as follows. The observed spectra and their reduction are described in Sect. 2. The NLTE calculations and spectrum synthesis are documented in Sect. 3. We present a considerable amount of details about the methods of calculation, because this is very important for a judgement of the resulting abundances. Methods of the abundance calculations and spectrum synthesis are described in Sect. 4, and the main results are summarized in Sect. 5. They include the run of $\mathrm{Mn} / \mathrm{Fe}$ abundance ratios with overall metallicity, a comparison with some recent LTE results for Mn abundances, and their relation to the chemical evolution of the Milky Way.

\section{Observations and stellar parameters}

Our sample consists of fourteen stars that were observed with the ESO UVES echelle spectrograph at the VLT UT2 on the 
Table 1. Stellar parameters and their estimated errors for the selected sample.

\begin{tabular}{|c|c|c|c|c|c|c|c|c|c|c|c|}
\hline \multirow[t]{2}{*}{ Object $^{a}$} & \multirow[t]{2}{*}{ HIP } & \multirow{2}{*}{$\begin{array}{l}T_{\text {eff }} \\
{[\mathrm{K}]}\end{array}$} & \multirow[t]{2}{*}{$\log g$} & \multirow{2}{*}{$\begin{array}{c}\xi_{\mathrm{t}} \\
{\left[\mathrm{km} \mathrm{s}^{-1}\right]}\end{array}$} & \multirow[t]{2}{*}[\mathrm{Fe}/\mathrm{H}]{} & \multirow[t]{2}{*}[\mathrm{Mg}/\mathrm{Fe}]{} & \multirow[t]{2}{*}{$\pi / \sigma_{\pi}$} & \multirow[t]{2}{*}{ Population } & \multirow[t]{2}{*}{$N^{b}$} & \multicolumn{2}{|c|}{$[\mathrm{Mn} / \mathrm{Fe}]$} \\
\hline & & & & & & & & & & NLTE & LTE \\
\hline HD & 594 & $85 \pm$ & \pm 0 & 1.5 & -1.96 & 0 & 22.7 & $\mathrm{H}$ & 10 & - & 0.52 \\
\hline $\mathrm{HD}$ & & 00 & 3 & & & & 1 & ? & 6 & 2 & \\
\hline HD & 60 & 0 & 9 & 0 . & 0 & 3 & 17.3 & $\mathrm{o} ?$ & 17 & 03 & 3 \\
\hline HD 3 & 24316 & $53 \pm 10$ & $4.47 \pm 0.07$ & 1. & 1.66 & 0.42 & 14.4 & Halo & 11 & $-0.10 \pm 0.02$ & $-0.5 /=$ \\
\hline HD 6 & 279 & $10 \pm$ & $3.96 \pm 0.05$ & 1. & 0.03 & 0.0 & 324.9 & Thin di & 17 & $-0.06 \pm 0.04$ & -0.08 \\
\hline HD & 15 & $b \pm$ & $4.00 \pm$ & 1. & 6 & 0.32 & 11.7 & $\mathrm{H}$ & 7 & \pm 0.02 & -0 \\
\hline $\mathrm{HD}$ & 360 & $20 \pm$ & $4.17 \pm$ & 1. & - & $t$ & .5 & & 11 & .04 & -( \\
\hline $\mathrm{HI}$ & 939 & $10 \pm$ & $4.69 \pm$ & 1 . & 5 & 6 & 140.0 & $\mathrm{H}$ & 10 & $-0.07 \pm$ & -0 \\
\hline BI & 109 & 0 - & 3 & 1. & 3 & 4 & 3.7 & & 4 & $2 \pm 0.00$ & 09 \\
\hline & & & & 1. & 78 & & 7.4 & $\mathrm{Ha}$ & 12 & 0.03 & -0.47 \\
\hline HD & 68594 & 00 & 1. & 1. & 51 & & 5.2 & $\mathrm{Ha}$ & 7 & $-0.15 \pm 0.05$ & -0.59 \\
\hline HD & 976 & $3 \pm 60$ & & 1 & & 43 & 18.0 & & 7 & $2 \pm 0.02$ & 0.10 \\
\hline G20 & & $5 \pm 80$ & $4.20 \pm 0.20$ & 1.5 & & & 5.1 & Halo & 2 & $-0.02 \pm 0.03$ & $-0.48 \pm 0.03$ \\
\hline HD 200580 & 103987 & $5940 \pm 80$ & $3.96 \pm 0.06$ & 1.4 & -0.82 & 0.46 & 13.8 & Thick disk & 10 & $+0.26 \pm 0.07$ & $+0.14 \pm 0.07$ \\
\hline
\end{tabular}

${ }^{a}$ An asterisk (*) marks the stars that were analyzed directly relative to the Sun. The Mn abundances of all other stars are from analyses relative to HD 102200, the results of which were added to the mean logarithmic abundance ratio $[\mathrm{Mn} / \mathrm{Fe}]$ of this star relative to the Sun.

${ }^{b}$ The number of Mn I lines used for individual stars.

Paranal, Chile, in 2001, and with the FOCES echelle spectrograph mounted on the $2.2 \mathrm{~m}$ telescope of the CAHA observatory on Calar Alto, during 1999 and 2000. For four stars (HD 61421, HD 84937, HD 140283, and BD-4³208), spectra obtained with both telescopes were available. The UVES spectra have a slit-determined resolution of $\lambda / \Delta \lambda \sim 50000$ and a signal-to-noise ratio better than $S / N \sim 300$ near $5000 \AA$. The data cover a spectral range from 3300 to $6700 \AA$, with a beam-splitter gap between 4470 and $4620 \AA$. For the other stars, either only UVES spectra (HD 29907, HD 34328, HD 102200, HD 122196) or FOCES spectra (HD 19445, HD 25329, HD 103095, HD 200580) were available. The latter have a resolution of $\sim 60000$ but an $S / N$ of only $\sim 200$ near $5000 \AA$, with the exception of G20-8, which was observed with a resolution of only $\sim 40000$. The UVES observations of Procyon (HD 61421) and HD 84937 were taken from the UVESPOP survey (Bagnulo et al. 2003). All stars on our list were observed at least twice. Table 1 lists the basic stellar parameters and the abundance results for our stars. The individual population membership is taken from Gehren et al. (2006), based on the $[\mathrm{Al} / \mathrm{Mg}]$ ratio and stellar kinematics. It is evident that most stars of the sample belong to the halo.

Parameters for most of the stars were taken from the analyses of Gehren et al. (2004, 2006), and Fuhrmann (2004). They were obtained using the Balmer line profile fits of $\mathrm{H}_{\alpha}$ and $\mathrm{H}_{\beta}$ for the determination of $T_{\text {eff }}$, a method quantified in Fuhrmann et al. (1993). The errors are obtained directly from profile fitting, and they are estimated to stay within $\pm 50 \ldots 100 \mathrm{~K}$ depending on a number of assumptions that add to systematic errors (e.g., line broadening theory, type of atmospheric models, treatment of convection). Generally, however, the method is the most reliable among all of the methods available for the temperature determination of solar-type stars. The calculation of the surface gravity acceleration $\log g$ is based on HIPPARCOS parallaxes and on masses estimated from tracks of stellar evolution kindly provided by VandenBerg et al. (2000) as indicated in Fuhrmann et al. (1997) or Gehren et al. (2004; 2006). Using the common symbol $[X]$ to denote the logarithmic value of a stellar parameter $X$ relative to the Sun, it follows that the errors propagate as $\Delta[g]=\Delta[M]+2 \Delta[\pi]+4 \Delta\left[T_{\text {eff }}\right]$ with some minor additional error from interstellar extinction and bolometric correction. Since the individual errors in mass, parallax and temperature are largely independent, the errors of $\log g$ for turnoff stars are caused almost entirely by the parallax errors, whereas errors in mass determination or temperature are usually at least a factor of 2 smaller, and those from reddening or bolometric correction are even less. Two exceptions to this rule are the cool main sequence stars HD 29907 and HD 103095, for which the mass determination is most uncertain. We note that the worst parallax of all stars in our sample is that of BD- $4^{\circ} 3208$ with $\sigma_{\pi} / \pi=0.27$; this makes that particular surface gravity uncertain by more than \pm 0.2 dex. The parameter $\pi / \sigma_{\pi}$ in Table 1 represents the inverse fractional parallax error. The iron abundance and microturbulence velocities are derived from fitting Fe II line profiles under the requirement that the Fe abundances are independent of line strengths. The uncertainties in $[\mathrm{Fe} / \mathrm{H}]$ and $\xi_{\mathrm{t}}$ are estimated to be 0.05 dex and $0.2 \mathrm{~km} \mathrm{~s}^{-1}$, respectively.

One and the same type of atmospheric model is used to determine stellar parameters and perform spectrum synthesis. The models are line-blanketed LTE atmospheres generated by the MAFAGS code (Fuhrmann et al. 1997). They use the opacity distribution functions of Kurucz (1992), and they reproduce the corresponding ATLAS9 models to within $<20 \mathrm{~K}$. To account for the high $\mathrm{Fe}$ abundance in these ODFs $\left(\log \varepsilon_{\mathrm{Fe}, \odot}=7.67\right)$, our models are scaled down in overall metal abundance by -0.16 dex. Since with the exception of Procyon our sample contains only thick disk and halo stars, the ODFs for the rest of the sample were additionally scaled for an $\alpha$-element overbundance of +0.4 dex, in rough agreement with previous analyses (Fuhrmann 2004; Gehren et al. 2006). A few of the stars are not easy to interpret. HD 25329 is nitrogen-rich, and HD 29907 is a single-lined binary. Both have an $[\mathrm{Al} / \mathrm{Mg}]$ ratio that is typical of the thick disk rather than of the halo, but both have $[\mathrm{Fe} / \mathrm{H}]$ abundances more typical of the Galactic halo. The asymmetrical drift velocity of HD 29907 deviates significantly from the average thick-disk velocity distribution, and its $[\mathrm{Eu} / \mathrm{Ba}]$ ratio is also typical of the halo (Mashonkina et al. 2003).

\section{Methods of NLTE calculations}

The NLTE level departure coefficients $b_{i}(\tau)$ are calculated using a Mn model atom similar to that described in Paper I; minor 

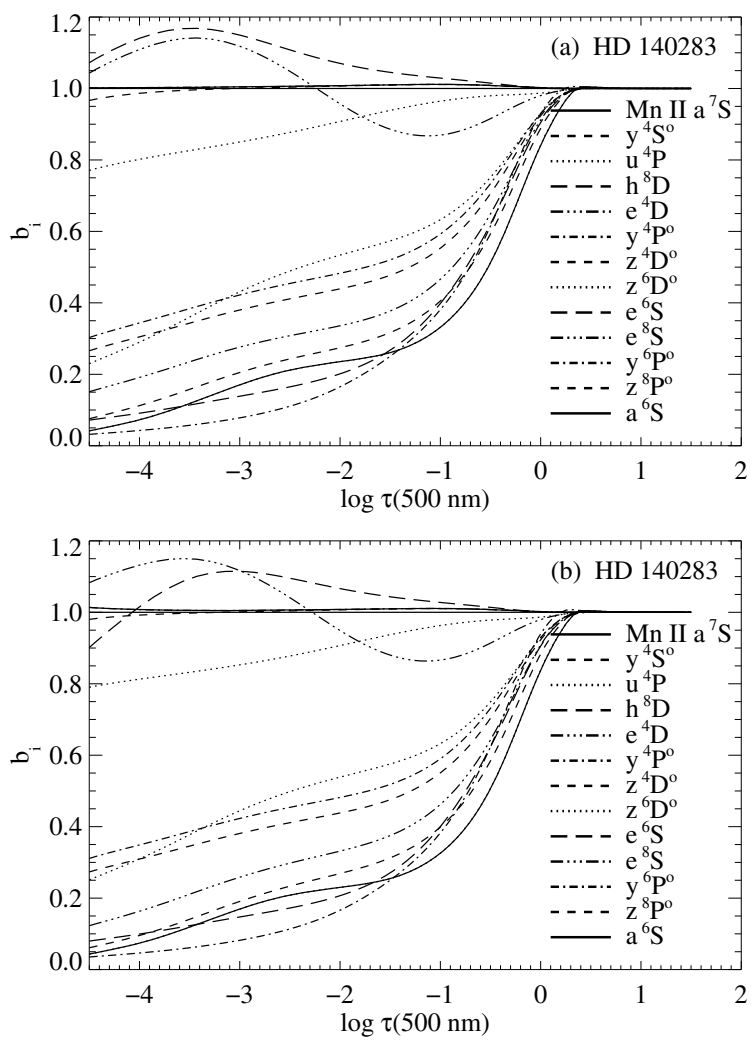

Fig. 1. Departure coefficients $b_{i}$ of selected Mn I levels for the metalpoor subgiant HD 140283: a) reduced model with 245 levels of Mn I and closed by the Mn II ground state; b) full model with 459 levels of Mn I, Mn II, and Mn III.

modifications in the model refer to the treatment of photoionization cross-sections and a number of levels. The code DETAIL (Butler \& Giddings 1985) based on the method of accelerated lambda iteration is used. We adopt the effective principal quantum numbers in calculation of photoionization cross-sections. The model is constructed with 245 levels of Mn I and closed by the ground state of Mn II; it is by a factor of two smaller than the model atom investigated in Paper I. Neglecting Mn II levels and Mn III ground state has almost no influence on the behavior of Mn I level departure coefficients. Figure 1 shows that departure coefficients for the levels of interest calculated for the most metal-poor star HD 140283 are identical over the whole optical depth scale. The difference in the models is only reflected in the behavior of the Mn II ground state and highly-excited Mn I levels in the outer atmosphere. The main advantage is that the time of computation is decreased significantly with the reduced atomic model.

We performed test calculations for selected models from a grid of theoretical atmospheres with the purpose of investigating NLTE effects for Mn I as a function of stellar parameters. In this range, $T_{\text {eff }}$ varies between 5000 and $6200 \mathrm{~K}, 3.0 \leq \log g \leq 4.6$, and $-3.0 \leq[\mathrm{Fe} / \mathrm{H}] \leq 0.0$. The microturbulent velocity $\xi_{\mathrm{t}}=1$ is assumed in all models. The inelastic collisions with hydrogen atoms were neglected in the test calculations $\left(S_{\mathrm{H}}=0\right)$. This results in slightly stronger NLTE effects compared to the case when hydrogen collisions are accounted for with our standard value $\left(S_{\mathrm{H}}=0.05\right)$. On the other hand, a simple hydrogenic approximation for photoionization underestimates the NLTE effects in Mn I (see a discussion in Paper I). As both assumptions work in opposite directions, we expect that our test models are representative of the true NLTE effects acting in Mn I. We show
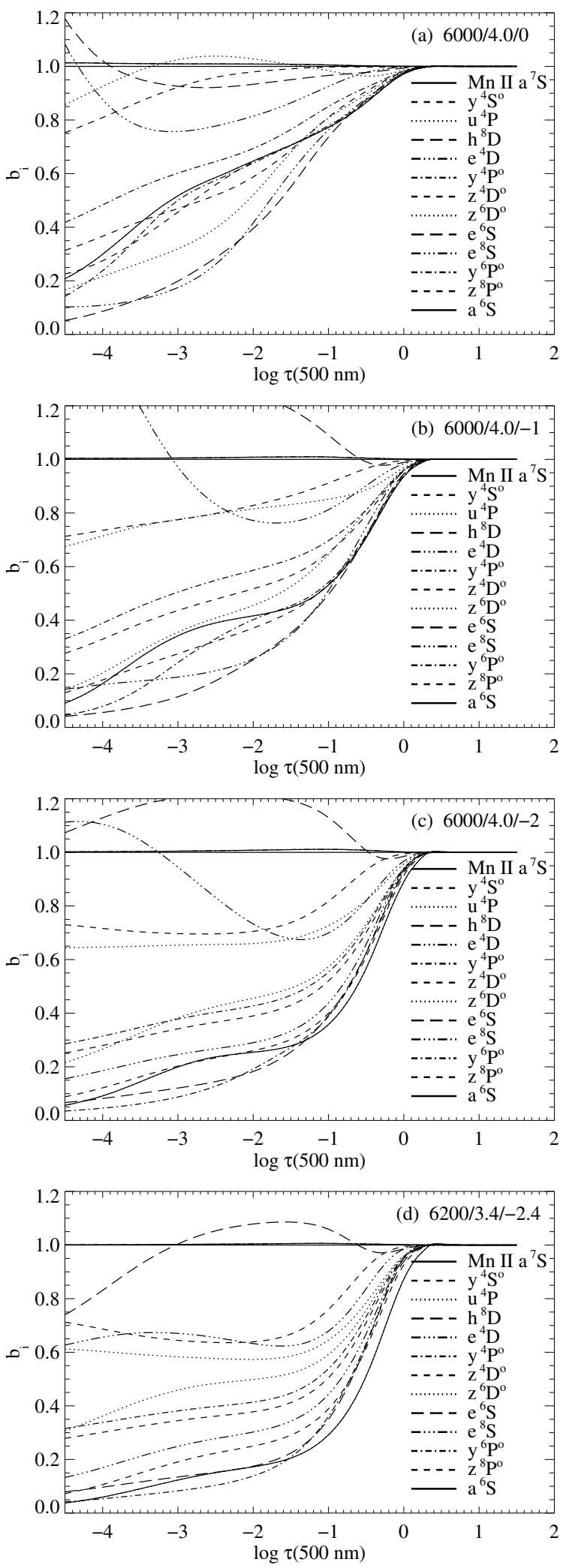

Fig. 2. Departure coefficients $b_{i}$ of selected Mn I levels for stellar models with different parameters.

in Sect. 5 how variation in $S_{\mathrm{H}}$ affects the abundances calculated from the line-profile fitting.

The departure coefficients $b_{i}$ computed for four models of the grid with different metallicities are shown in Fig. 2. All Mn I levels involved in transitions of interest are underpopulated above 
Table 2. NLTE abundance corrections computed with $S_{\mathrm{H}}=0$ for selected models of the grid.

\begin{tabular}{|c|c|c|c|c|c|c|c|c|c|c|c|c|c|c|c|c|c|c|}
\hline \multirow{2}{*}{$\begin{array}{r}T_{\text {eff }} / \log g / \\
{[\mathrm{Fe} / \mathrm{H}]}\end{array}$} & \multicolumn{18}{|c|}{$\Delta_{\text {NLTE }}$} \\
\hline & 4018 & 4030 & 4033 & 4034 & 4041 & 4055 & 4451 & 4739 & 4754 & 4761 & 4762 & 4765 & 4766 & 4783 & 4823 & 6013 & 6016 & 6021 \\
\hline $5000 / 4 / 0$ & 0.03 & 0.05 & 0.05 & 0.05 & 0.04 & 0.04 & 0.03 & 0.04 & 0.02 & 0.03 & 0.03 & 0.04 & 0.03 & 0.03 & 0.04 & -0.09 & -0.1 & -0.08 \\
\hline $5000 / 4 /-1$ & 0.15 & 0.15 & 0.15 & 0.15 & 0.14 & 0.15 & 0.2 & 0.24 & 0.09 & 0.25 & 0.18 & 0.24 & 0.24 & 0.13 & 0.12 & 0.17 & 0.16 & 0.13 \\
\hline $5000 / 4 /-2$ & 0.47 & 0.23 & 0.24 & 0.24 & 0.42 & 0.49 & 0.43 & - & 0.36 & - & 0.39 & 0.41 & 0.42 & 0.35 & 0.34 & - & - & - \\
\hline $5000 / 4 /-3$ & 0.54 & 0.45 & 0.45 & 0.49 & 0.56 & - & - & - & 0.48 & - & - & - & - & 0.5 & 0.49 & - & - & - \\
\hline $5500 / 4 / 0$ & 0.05 & 0.08 & 0.08 & 0.08 & 0.05 & 0.02 & 0.05 & 0.1 & 0.05 & 0.1 & 0.05 & 0.1 & 0.06 & -0.01 & -0.01 & 0.05 & 0.04 & 0.01 \\
\hline $5500 / 4 /-1$ & 0.28 & 0.1 & 0.15 & 0.15 & 0.26 & 0.25 & 0.26 & 0.27 & 0.15 & 0.29 & 0.22 & 0.3 & 0.29 & 0.14 & 0.14 & 0.24 & 0.24 & 0.22 \\
\hline $\mid-2$ & 0.42 & 0.28 & 0.31 & 0.35 & 0.48 & 0.43 & 0.39 & - & 0.38 & - & 0.38 & 0.38 & 0.38 & 0.4 & 0.4 & - & - & - \\
\hline $5500 / 4 /-3$ & 0.51 & 0.69 & 0.7 & 0.72 & 0.51 & - & - & - & 0.5 & - & - & - & - & 0.52 & 0.52 & - & - & - \\
\hline $6000 / 4 / 0$ & 0.1 & 0.07 & 0.07 & 0.08 & 0.05 & -0.02 & 0.05 & 0.11 & -0.02 & 0.11 & 0.05 & 0.1 & 0.08 & -0.03 & -0.04 & 0.06 & 0.05 & 0.01 \\
\hline $6000 / 4 /-1$ & 0.28 & 0.1 & 0.1 & 0.1 & 0.27 & 0.29 & 0.26 & 0.26 & 0.19 & 0.27 & 0.22 & 0.3 & 0.3 & 0.17 & 0.15 & 0.22 & 0.23 & 0.22 \\
\hline $6000 / 4 /-2$ & 0.38 & 0.4 & 0.42 & 0.42 & 0.38 & 0.41 & 0.36 & - & 0.35 & - & 0.34 & 0.35 & 0.35 & 0.37 & 0.37 & - & - & - \\
\hline $6000 / 4 /-3$ & - & 0.63 & 0.64 & 0.64 & 0.4 & - & - & - & 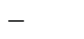 & - & - & - & - & 0.42 & 0.42 & - & - & - \\
\hline $6200 / 3.4 / 0$ & 0.03 & 0.06 & 0.07 & 0.07 & 0.01 & 0.02 & 0.01 & 0.1 & -0.07 & 0.09 & 0 & 0.06 & 0.04 & -0.07 & -0.07 & 0 & -0.03 & -0.07 \\
\hline $6200 / 3.4 /-1.2$ & 0.32 & 0.1 & 0.1 & 0.15 & 0.31 & 0.38 & 0.32 & 0.28 & 0.27 & 0.28 & 0.28 & 0.31 & 0.31 & 0.25 & 0.25 & 0.25 & 0.24 & 0.25 \\
\hline $6200 / 3.4 /-2.4$ & 0.4 & 0.55 & 0.6 & 0.63 & 0.4 & 0.41 & 0.36 & - & 0.36 & - & 0.35 & 0.35 & 0.36 & 0.39 & 0.4 & - & - & - \\
\hline $6200 / 4.6 / 0$ & 0.05 & 0.08 & 0.08 & 0.07 & 0.02 & 0.03 & 0.03 & 0.1 & -0.01 & 0.08 & 0.04 & 0.06 & 0.04 & 0.01 & 0.02 & 0.02 & 0 & -0.03 \\
\hline $6200 / 4.6 /-1.2$ & 0.28 & 0.15 & 0.15 & 0.15 & 0.26 & 0.31 & 0.28 & 0.26 & 0.24 & 0.26 & 0.25 & 0.28 & 0.28 & 0.23 & 0.22 & 0.23 & 0.23 & 0.23 \\
\hline $6200 / 4.6 /-2.4$ & 0.38 & 0.43 & 0.47 & 0.51 & 0.39 & 0.39 & 0.35 & - & 0.35 & - & 0.34 & 0.34 & 0.34 & 0.36 & 0.36 & - & - & - \\
\hline
\end{tabular}

$\log \tau_{5000} \approx 0$. This is characteristic of all models irrespective of the temperature, gravity, and metallicity. Hence the NLTE assumption generally leads to an increase in the Mn I line formation depths that forces a weakening of the lines.

The NLTE effects become more pronounced with increasing effective temperature and decreasing metallicity. In both cases, the UV radiation field is amplified and increases the rates of $b-b$ and $\mathrm{b}-\mathrm{f}$ transitions in the MnI atom. However, metallicity has a stronger influence on the populations of levels of interest than effective temperature. The reason is that with increasing temperature the collision rates also increase due to a larger kinetic energy of the particles. This effect counteracts the NLTE influence of the radiation field. Whereas decreased metallicity implies a lower number density of metals, which are the major suppliers of free electrons. As a result, the reduced continuous opacity in the UV and decreased collision rates result in a strong depopulation of all Mn I levels.

In the solar metallicity model (Fig. 2a), radiative transitions with strong optical pumping effects are largely responsible for depopulation of the low and intermediate energy levels. Photoionization from these levels is inefficient, and that is the result of too low cross-section approximations (see discussion in Paper I). In the metal-poor models (Figs. 2b and c), the dominant NLTE process is overionization from the low-excitation levels $\mathrm{a}^{6} \mathrm{D}, z^{8} \mathrm{P}^{\circ}$ and $\mathrm{a}^{4} \mathrm{D}^{\circ}$ with thresholds at $\lambda \sim 2330 \AA, \lambda \sim 2406 \AA$, and $\lambda \sim 2727 \AA$, respectively. Radiative b-b transition rates can no longer compete with photoionization.

Variation of gravity has a marginal effect on the atomic level populations. For the whole range of stellar parameters considered here, the change in gravity from $\log g=4.6$ to $\log g=3.0$ results in a slightly weaker coupling of the levels with each other. This is the result of a decreased collision frequency, which tends to destroy LTE population ratios between the levels. We show below that only at $[\mathrm{Fe} / \mathrm{H}] \leq-2$ and $T_{\text {eff }} \geq 6000 \mathrm{~K}$ is the population of the Mn I ground state is appreciably affected by the change of gravity.

\subsection{NLTE and HFS effects on spectral lines and Mn abundances}

The abundance corrections $\Delta_{\text {NLTE }}$ required to equalize the NLTE and LTE equivalent widths of the Mn I lines are given in Tables 2 and 3. The values in Table 2 are calculated with the reference model of the Mn atom, but setting the scaling factor for hydrogen collisions to 0 . Table 3 shows the NLTE abundance corrections for the same models calculated with $S_{\mathrm{H}}=0.05$. Hyphens refer to the lines with theoretical NLTE equivalent widths below $3 \mathrm{~mA}$. We set this lower limit to the calculated $W_{\lambda}$ because our spectra do not have a sufficient signal-to-noise ratio to measure the abundances from such weak lines with an accuracy of less than $10 \%$. For clarity, the discussion below is based on the data from Table 2 calculated with $S_{\mathrm{H}}=0$. Apart from the guesswork that collisions with neutral hydrogen atoms may become a main thermalizing mechanism in metal-poor stars, we know nothing about them.

The NLTE effects on a Mn I line at $4033 \AA$ for selected stellar models from the grid are shown in Fig. 4a. As in the previous section, we select three models with different metallicities for comparison. Other model parameteres are equal: $T_{\text {eff }}=6000 \mathrm{~K}$ and $\log g=4$.

The NLTE mechanisms, responsible for the behavior of line profiles at a solar metallicity, were explained in Paper I in detail. The strong lines are characterized by an amplified absorption in the core and a decreased absorption in the wings relative to LTE. The physics behind it is the overionization at the depth of the line-wing formation, where $b_{i}<1$ and $b_{i}<b_{j}$. At the depths of the core formation, photon losses in the line wings result in $b_{i}>b_{j}$, so the core in NLTE is deeper than in LTE. The effect on the abundances is relatively small. As can be seen in Table 2, the NLTE abundance corrections $\Delta_{\text {NLTE }}$ vary from +0.1 to -0.1 dex for any model with $[\mathrm{Fe} / \mathrm{H}]=0$.

With decreasing metallicity, line formation shifts to deeper layers, where photoionization is dominant in depopulating all Mn I levels. Maximum $\Delta_{\text {NLTE }}$ are found for the excited lines in the models with $T_{\text {eff }} \leq 5500 \mathrm{~K}$ and $[\mathrm{Fe} / \mathrm{H}] \geq-2$.

With increasing $T_{\text {eff }}$, the stellar flux maximum is shifted to the shorter wavelengths, and ionization from the ground state of Mn I becomes more important. In the models with $T_{\text {eff }} \geq 6000 \mathrm{~K}$ and $[\mathrm{Fe} / \mathrm{H}] \leq-2$, the NLTE abundance effect is more pronounced for the resonance triplet at $403 \mathrm{~nm}$. However, as emphasized above, increasing $T_{\text {eff }}$ also increases the rates of collisions, so maximum NLTE effects for the resonance lines are in fact found for the moderately warm model $\left(T_{\text {eff }} \geq 5500\right)$ with lowest metallicity (least amount of free electrons). 
Table 3. NLTE abundance corrections computed with $S_{\mathrm{H}}=0.05$ for selected models of the grid.

\begin{tabular}{|c|c|c|c|c|c|c|c|c|c|c|c|c|c|c|c|c|c|c|}
\hline \multirow{2}{*}{$\begin{array}{r}T_{\text {eff }} / \log g / \\
{[\mathrm{Fe} / \mathrm{H}]}\end{array}$} & \multicolumn{18}{|c|}{$\bar{\Delta}_{\mathrm{NLTE}}$} \\
\hline & 4018 & 4030 & 4033 & 4034 & 4041 & 4055 & 4451 & 4739 & 4754 & 4761 & 4762 & 4765 & 4766 & 4783 & 4823 & 6013 & 6016 & 6021 \\
\hline $5000 / 4 / 0$ & 0.03 & 0.04 & 0.04 & 0.05 & 0.03 & 0.03 & 0.03 & 0.01 & 0.02 & 0.01 & 0.02 & 0.02 & 0.02 & 0.02 & 0.02 & -0.08 & -0.09 & -0.09 \\
\hline $5000 / 4 /-1$ & 0.15 & 0.1 & 0.1 & 0.1 & 0.13 & 0.11 & 0.15 & 0.19 & 0.07 & 0.2 & 0.13 & 0.2 & 0.17 & 0.07 & 0.07 & 0.12 & 0.1 & 0.07 \\
\hline $5000 / 4 /-2$ & 0.41 & 0.23 & 0.23 & 0.23 & 0.36 & 0.43 & 0.37 & - & 0.31 & - & 0.34 & 0.37 & 0.37 & 0.3 & 0.3 & - & - & - \\
\hline $5000 / 4 /-3$ & 0.49 & 0.37 & 0.37 & 0.41 & 0.5 & - & - & - & 0.42 & - & - & - & - & 0.45 & 0.44 & - & - & - \\
\hline $5500 / 4 / 0$ & 0.02 & 0.05 & 0.04 & 0.04 & 0.02 & 0.03 & 0.01 & 0.04 & 0 & 0.03 & 0 & 0.03 & 0.01 & 0.01 & 0.01 & -0.08 & -0.12 & -0.1 \\
\hline $5500 / 4 /-1$ & 0 & 0.09 & 0.13 & 0.14 & 0.15 & 0.15 & 0.2 & 0.23 & 0.07 & 0.24 & 0.16 & 0.24 & 0.23 & 0.07 & 0.07 & 0.17 & 0.16 & 0.15 \\
\hline $5500 / 4 /-2$ & 0.41 & 0.27 & 0.28 & 0.29 & 0.42 & 0.39 & 0.42 & - & 0.33 & - & 0.33 & 0.34 & 0.34 & 0.35 & 0.35 & - & - & - \\
\hline $5500 / 4 /-3$ & 0.46 & 0.59 & 0.6 & 0.61 & 0.45 & 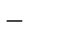 & - & - & 0.44 & - & - & - & 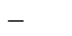 & 0.47 & 0.47 & - & - & - \\
\hline $6000 / 4 / 0$ & 0.02 & 0.05 & 0.05 & 0.05 & 0.01 & 0.01 & 0.01 & 0.06 & -0.04 & 0.04 & 0.01 & 0.03 & 0.02 & -0.02 & -0.02 & -0.02 & -0.06 & -0.08 \\
\hline $6000 / 4 /-1$ & 0.21 & 0.11 & 0.1 & 0.11 & 0.18 & 0.21 & 0.2 & 0.2 & 0.13 & 0.21 & 0.17 & 0.22 & 0.22 & 0.1 & 0.1 & 0.16 & 0.16 & 0.17 \\
\hline $6000 / 4 /-2$ & 0.35 & 0.3 & 0.3 & 0.33 & 0.34 & 0.37 & 0.31 & - & 0.3 & - & 0.3 & 0.31 & 0.31 & 0.33 & 0.33 & - & - & - \\
\hline $6000 / 4 /-3$ & - & 0.6 & 0.61 & 0.61 & 0.38 & - & - & - & - & - & - & - & - & 0.38 & 0.39 & - & - & - \\
\hline $6200 / 3.4 / 0$ & 0.01 & 0.05 & 0.06 & 0.07 & -0.03 & 0.01 & 0 & 0.08 & -0.1 & 0.07 & 0 & 0.07 & 0.03 & -0.08 & -0.1 & 0 & -0.03 & -0.07 \\
\hline $6200 / 3.4 /-1.2$ & 0.27 & 0.05 & 0.06 & 0.11 & 0.26 & 0.32 & 0.26 & 0.24 & 0.22 & 0.24 & 0.22 & 0.25 & 0.26 & 0.2 & 0.2 & 0.2 & 0.19 & 0.2 \\
\hline $6200 / 3.4 /-2.4$ & 0.35 & 0.5 & 0.52 & 0.56 & 0.35 & 0.37 & 0.32 & - & 0.31 & - & 0.31 & 0.31 & 0.31 & 0.34 & 0.34 & - & - & - \\
\hline $6200 / 4.6 / 0$ & 0.01 & 0.04 & 0.05 & 0.05 & 0.05 & 0.01 & 0.01 & 0.06 & -0.02 & 0.04 & 0.02 & 0.04 & 0.02 & -0.01 & -0.02 & 0 & -0.02 & -0.04 \\
\hline $6200 / 4.6 /-1.2$ & 0.22 & 0.1 & 0.1 & 0.11 & 0.2 & 0.25 & 0.22 & 0.21 & 0.18 & 0.21 & 0.19 & 0.22 & 0.22 & 0.17 & 0.16 & 0.17 & 0.17 & 0.17 \\
\hline $6200 / 4.6 /-2.4$ & 0.34 & 0.37 & 0.39 & 0.43 & 0.34 & 0.35 & 0.3 & - & 0.31 & - & 0.30 & 0.3 & 0.3 & 0.33 & 0.33 & - & - & - \\
\hline
\end{tabular}

It is remarkable that, for all models with very low metal abundance except $T_{\text {eff }} \sim 5000 \mathrm{~K}$, we find a difference of $\sim+0.2$ dex between the NLTE corrections for resonance and excited lines. This difference is enhanced for the model with lower gravity $(\log g=3.4)$. Thus, NLTE can solve the discrepancy between the abundances derived from the Mn I resonance triplet at $403 \mathrm{~nm}$ and excited lines, which is found in analyses of metal-poor subdwarfs and subgiants (Gratton 1987; Gratton 1989; Lai et al. 2008). The test calculations for the cool giant model $\left(T_{\text {eff }}=4800 \mathrm{~K}, \log g=1.8,[\mathrm{Fe} / \mathrm{H}]=-3.3\right.$, not shown in Table 2) also indicate that the NLTE corrections for the resonance lines are by $\sim 0.15$ dex higher than those for excited lines at 4783 and $4823 \AA$. This is the first proof that the discrepancies between two line sets found by Johnson (2002) and Cayrel et al. (2004) in their studies of giant stars are due to NLTE.

It is useful to inspect the sensitivity of NLTE abundance corrections $\Delta_{\text {NLTE }}$ to the treatment of inelastic collisions with hydrogen. This is a factor that can produce systematic errors in NLTE calculations. In the following sections, we will show how the differential $[\mathrm{Mn} / \mathrm{Fe}]$ ratios for the program stars react to changes in $S_{\mathrm{H}}$. Here, we instead concentrate on the change in NLTE abundance corrections derived for individual lines for several models from the grid. Figure 3 demonstrates the behaviour of $\Delta_{\text {NLTE }}$ calculated with three different scaling factors to the standard Drawins formula 0,0.05, 1 (solid, dashed, and dotted lines, respectively) as a function of a model metallicity $\left(T_{\text {eff }}=6000 \mathrm{~K}\right.$ and $\left.\log g=4\right)$. Here, the average $\Delta_{\text {NLTE }}$ derived for the resonance triplet at $403 \mathrm{~nm}$ are shown in the top panel, and that for the excited lines at 4783 and 4823 in the bottom panel. These five lines are traditionally used in the analyses of $\mathrm{Mn}$ in metal-poor stars. The resonance lines are weakly sensitive to $\mathrm{H}$ collisions at all metallicities, except for $[\mathrm{Fe} / \mathrm{H}] \sim-2$. But in the latter case, $\Delta_{\text {NLTE }}$ is already large enough. The excited lines steadily decrease in sensitivity to $\mathrm{H}$ collisions for decreasing Fe abundance. This is expected because the major overionization in Mn I is expected to occur from the low-excited levels, e.g., $z^{8} \mathrm{P}^{\mathrm{o}}$, but not from the ground state (see Paper I). In the metal-poor stars, overionization is strongly amplified, as is obvious from the magnitude of NLTE corrections thus reducing the impact of collisions on level populations. The important result following from Fig. 3 is that any collision scaling factor chosen within the reasonable range (see below) leads to a qualitatively similar behavior of NLTE abundance corrections. As a result, the error introduced by our poor knowledge of $S_{\mathrm{H}}$ is systematic, much like the errors in stellar parameters and models. With a free choice of $S_{\mathrm{H}}$ from 0 to 1, one would most likely choose the value that produces the NLTE abundance corrections lying in the middle of the upper and lower limits. In this respect, $S_{\mathrm{H}}=0.05$ (our reference value) seems to be a good choice. We show below that this value is also supported by the smallest fitted-abundance spread between different lines of selected stars.

We have also checked the influence of hyperfine splitting (HFS) on Mn I line formation. Figure 4b shows NLTE profiles of Mn I line at $4823 \AA$ calculated for three models with different metallicities $\left([\mathrm{Fe} / \mathrm{H}]=0,-1,-2, T_{\text {eff }}=5500 \mathrm{~K}, \log g=4\right)$. The synthetic profiles computed with and without HFS are marked with solid and dotted lines, respectively. The line is split into six HFS components. In the solar metallicity model, the profile computed without HFS overestimates the abundance by 0.35 dex. In the models with $[\mathrm{Fe} / \mathrm{H}]=-1$ and -2 , the difference in profiles corresponds to an abundance correction of 0.08 dex and 0.01 dex, respectively. This example demonstrates the significance of HFS effects, which must be treated correctly even when the abundance analysis of metal-poor stars is performed.

\section{Methods of abundance calculations}

\subsection{Spectrum synthesis}

Spectrum synthesis is carried out with the code SIU using the computed departure coefficients $b_{i}(\tau)$ for Mn I levels. For all other elements, LTE line formation is assumed. The stellar line profiles are convolved with a Gaussian of $\sim 3-4 \mathrm{~km} \mathrm{~s}^{-1}$. Rotational broadening and macroturbulence are only treated separately for Procyon. Van der Waals damping constants $\log C_{6}$ were determined in Paper I from solar line profile fitting. They are on average -0.1 dex lower than the values calculated according to the Anstee \& O'Mara (1995) line broadening tables. Multiplet 22 with lines at 4451, 4453, and $4502 \AA$ was calculated with $\Delta \log C_{6}=-0.6$ relative to the Anstee \& O'Mara values. This correction was necessary to make the Mn abundances derived from different lines of the multiplet agree with each other.

Solar $\log (g f \varepsilon)_{\odot}$ values used here are slightly different from those published in Bergemann \& Gehren (2007) and 

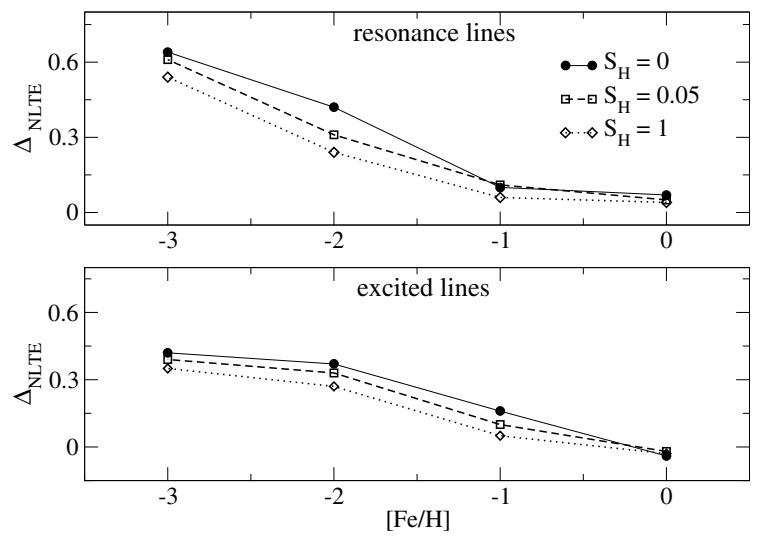

Fig. 3. NLTE abundance corrections $\Delta_{\text {NLTE }}$ (see text) calculated with three different values of a scaling factor to inelastic collisions with hydrogen $S_{\mathrm{H}}=0,0.05,1$ : the average for the resonance triplet at $403 \mathrm{~nm}$ (top), the average for excited lines at 4783 and $4823 \AA$. Calculations were performed for four models with $T_{\text {eff }}=6000, \log g=4,[\mathrm{Fe} / \mathrm{H}]=$ $0,-1,-2,-3$.
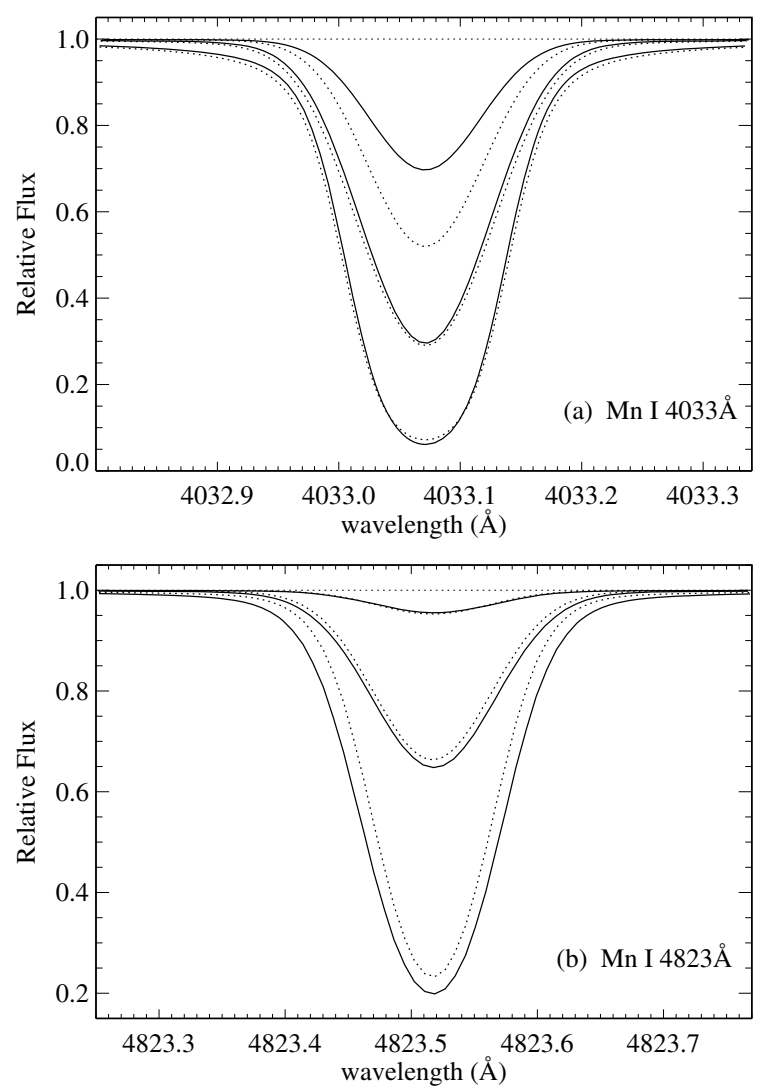

Fig. 4. Synthetic NLTE profiles of Mn I lines for different models from the grid. a) NLTE effects on the line at $4033 \AA$ for the models with constant effective temperature and gravity $\left(T_{\text {eff }}=6000, \log g=4\right)$ and different metallicities $([\mathrm{Fe} / \mathrm{H}]=0,-1,-2)$. b) HFS effects on the line at $4823 \AA$ for the models with $T_{\text {eff }}=5500, \log g=4,[\mathrm{Fe} / \mathrm{H}]=0,-1,-2$ (bottom to top).

Blackwell-Whitehead \& Bergemann (2007). This is due to an error in the treatment of hyperfine splitting, which we found in our previous analyses. This error led to a relatively large abundance scatter between different lines; however, the average NLTE abundance of Mn remained the same, $\log \varepsilon=5.37 \pm 0.06$ dex.

The resonance triplet at $403 \mathrm{~nm}$ was calculated with $\log C_{6}=$ -31.2 dex, that is, 0.65 dex higher than the values predicted by
Table 4. Lines of Mn I selected for abundance calculations.

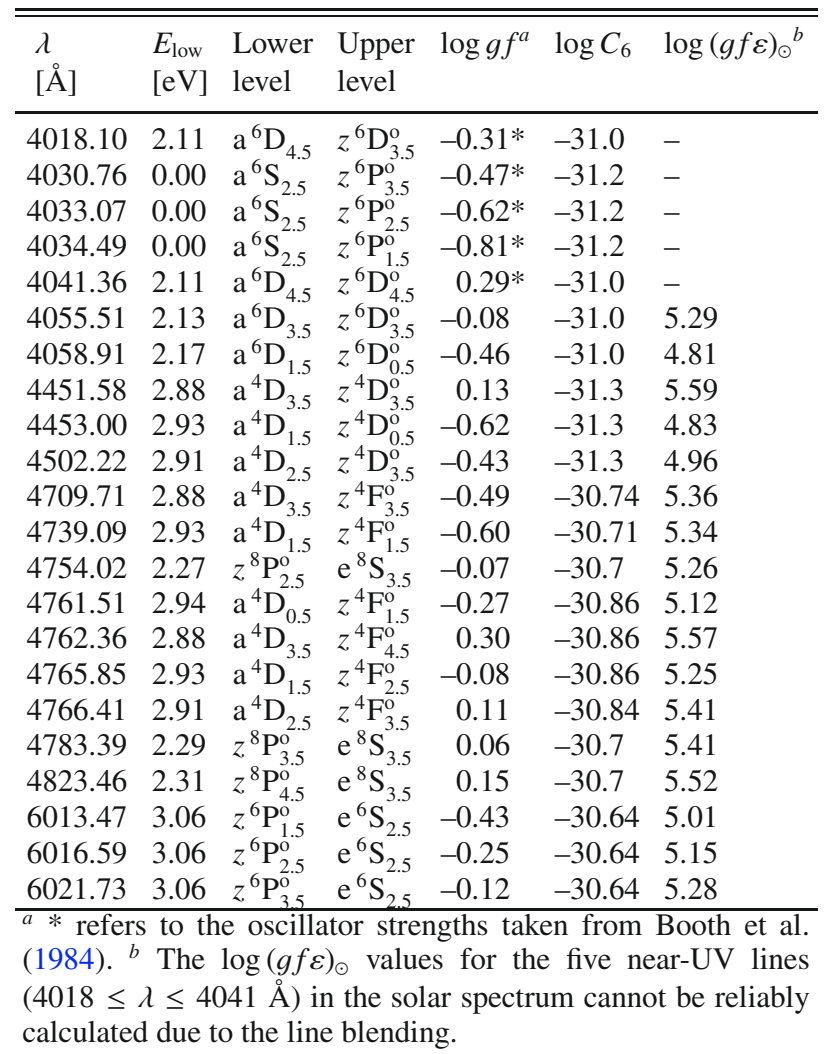

the Anstee \& O'Mara theory. Also, lower values of microturbulent velocity are required to fit these lines in the solar spectrum in contrast to all other lines, which are well-fitted with $\xi_{\mathrm{t}}=0.9 \mathrm{~km} \mathrm{~s}^{-1}$. This is a result of huge line strengths; e.g., the line at $4030 \AA$ has an equivalent width $W_{\lambda} \sim 325 \mathrm{~m} \AA$. Such strong lines are formed over a large optical depth interval, and their profiles reflect the depth-dependent velocity distribution. The cores are formed above $\log \tau_{5000} \sim-4.5$ being influenced by the chromosphere. In this particular case, the NLTE theory produces cores that are too narrow and deep compared to observations.

The line data used for spectrum synthesis are given in Table 4. Unless otherwise noted, the oscillator strengths are from Blackwell-Whitehead \& Bergemann (2007). The values of microturbulence velocities are listed in Table 1.

\subsection{Some aspects of a differential stellar analysis}

As evident from our previous studies (Bergemann \& Gehren 2007), the scatter among laboratory $g f$-values does not allow an accurate determination of the solar Mn abundance. Hence, we perform a differential analysis of stars with respect to the Sun ${ }^{1}$, which excludes the use of absolute oscillator strengths, but requires the knowledge of the $\log (g f \varepsilon)$ values for each line under investigation. The Mn abundance in a metal-poor star relative to the Sun is given by

$[\mathrm{Mn} / \mathrm{H}]=\log (g f \varepsilon)_{*}-\log (g f \varepsilon)_{\odot}$.

Five strong near-UV lines (4018 $\leq \lambda \leq 4041 \AA$ ) of Mn I cannot be used used for a reliable abundance analysis in the solar

\footnotetext{
${ }^{1}$ Every single abundance for a line fit in a stellar spectrum is related to the solar abundance from the same line.
} 
spectrum due to a severe blending. Unfortunately, in our spectra of the metal-poor stars, only these lines and three lines of multiplet 16 are detected. Hence, we define another "reference" star, HD 102200, with $[\mathrm{Fe} / \mathrm{H}]=-1.28 \mathrm{dex}$, whose $\log (g f \varepsilon)_{\text {ref }}$ values are used for the differential comparison with the more metal-poor stars. In this case, we calculate an average $[\mathrm{Mn} / \mathrm{H}]$ from all detected lines in spectrum of a metal-poor star relative to HD 102200 , and then correct this value by an average $[\mathrm{Mn} / \mathrm{H}]$ derived from the visual and near-IR lines of HD 102200 relative to the Sun.

Obviously, our analysis relies on the assumption that the relative NLTE abundances $[\mathrm{Mn} / \mathrm{H}]$ deduced from near-UV lines of HD 102200 do not deviate from the abundances deduced from the other lines of this star. Usually, the large scatter results from the incorrectly treated line blends, which have a different dependence on the $T_{\text {eff }}$ and $\log g$. Incorrect stellar parameters, e.g., microturbulence and model structure, may decrease the accuracy of the abundances. This problem can be partly solved by using the ratio of two similar elements $[\mathrm{Mn} / \mathrm{Fe}]$, since the same atmospheric models were used to obtain iron abundances in the stars studied here (see description in Sect. 2).

We have checked how blends contained in the near-UV Mn I lines affect the line profiles and $\mathrm{Mn}$ abundances derived from these lines in the Sun and in metal-poor stars. Figure 5 shows the line at $4030 \AA$ in the spectra of the Sun, HD 102200, and HD 19445, respectively. Vertical lines mark the Fe I blends in the red and blue wings. A solid line represents the best theoretical NLTE profile, the dotted line shows the same profile with $\log g f$ for both Fe I blends increased by 0.1 dex. Even this small correction produces a poor fit of the Mn I line wings in the solar spectrum; hence, we constrain the solar $\log g f$ values for the iron blends at 4030.5 and $4030.9 \AA$ to -0.8 and -1.6 dex, respectively. The uncertainty of these values is 0.05 dex. The profile of the same Mn I line in a metal-poor star is practically insensitive to a variation in iron line strengths. We, thus, expect that the relative $\mathrm{Mn}$ abundances derived from the near-UV lines in metal-poor stars are reliable.

It is noteworthy that the absolute LTE and NLTE abundances from the resonance triplet at $403 \mathrm{~nm}$ are lower than the abundances from the other lines on average by $0.07-0.2$ dex. This is a characteristic of all stars in our sample. In the differential LTE analysis relative to HD 102200, this inconsistency also appears and increases with decreasing metallicity. For the metalpoor star HD 140283, we infer the $\Delta[\mathrm{Mn} / \mathrm{H}]_{\text {LTE }}$ between the resonance and excited lines to be $\sim 0.3 \mathrm{dex}$. This finding supports the results of Cayrel et al. (2004). In their LTE analysis of Mn in metal-poor stars there is a systematic difference of $\sim 0.4$ dex between the near-UV (resonance triplet at $403 \mathrm{~nm}$ ) and visual Mn I lines (4754 and $4823 \AA$ ).

The discrepancy is also present in a differential NLTE analysis, but even in the most metal-poor stars it does not exceed 0.05 dex. We have good reason to believe that the problem is concealed in the van der Waals damping constants; namely, when the resonance lines are calculated with $\log C_{6}=-30.5$, the abundance differences between resonance and excited lines completely vanish for all stars in our sample. However, such a strong damping is cannot be applied to fitting the solar resonance lines. The wings are too broad, and that discrepancy cannot be removed by adjusting any other parameter in line synthesis (see upper panel of Fig. 4). Because of this, we decided to preserve the $\log C_{6}=-31.2$ for the resonance lines, keeping in mind the possible error of 0.05 dex introduced by the uncertain $\log C_{6}$ value.
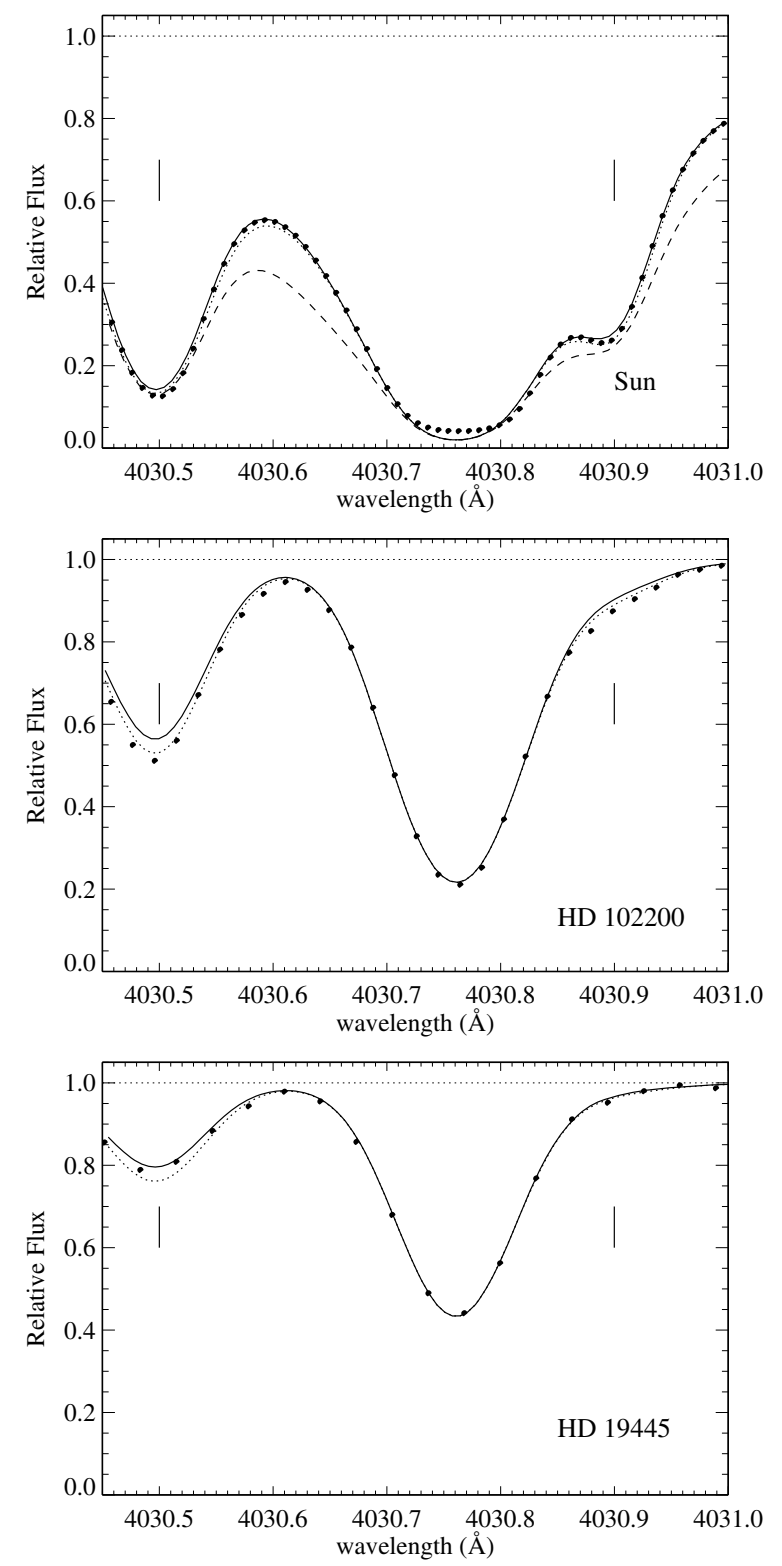

Fig. 5. The Fe I blends in the blue and red wings of the Mn I line at $4030 \AA$ in spectra of the Sun, HD 102200, and HD 19445 (from top to bottom). The best theoretical NLTE profile calculated with $\log C_{6}=$ -31.2 is marked with a solid line, the same profile with $\log g f$ for both Fe I blends increased by 0.1 dex is shown with a dotted line. The dashed line on the upper panel corresponds to the NLTE profile calculated with $\log C_{6}=-30.5$.

The problem elucidated above stems from the different sensitivities of strong and weak lines to the van der Waals damping. A weak line in a metal-poor star is strong in the solar spectrum, so its $\log (g f \varepsilon)$ value will depend on the adopted $C_{6}$ constant.

The most likely explanation for the discrepancy in absolute line abundances is that the oscillator strengths are erroneous. We use different sets of experimental $g f$-values for the near-UV and visual lines, those of Booth et al. (1984) and of BlackwellWhitehead \& Bergemann (2007), respectively. Cayrel uses the data of Booth et al. (1984). The comparison of Booth's values with the recent experiment of Blackwell-Whitehead revealed significant disagreement for several strong Mn I transitions.

Finally, a differential analysis requires similar modeling of microscale velocities in the Sun and stars. In metal-poor stars, 


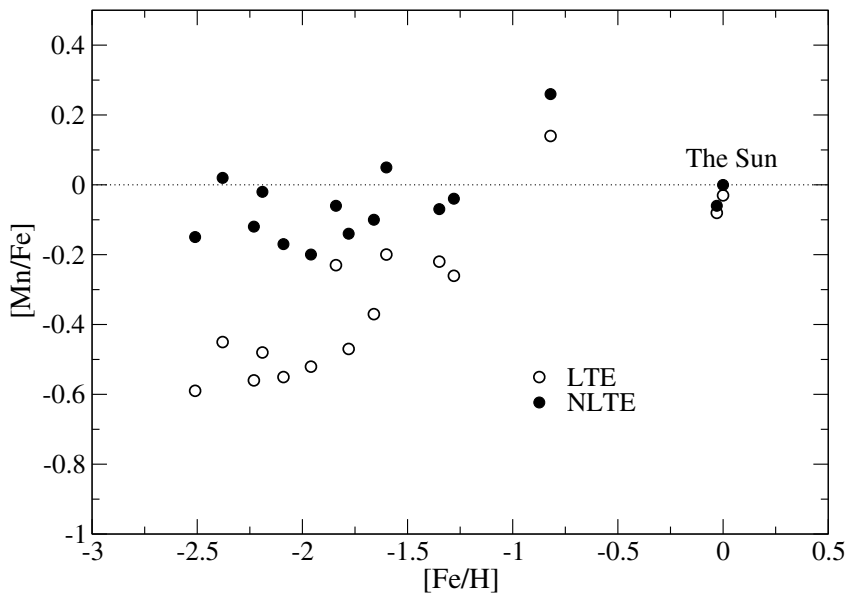

Fig. 6. $[\mathrm{Mn} / \mathrm{Fe}]$ vs. $[\mathrm{Fe} / \mathrm{H}]$ in metal-poor stars. NLTE abundances are marked with filled circles. LTE abundances are marked with empty squares. The spectroscopic binaries are shown with open circles.

only a single depth-independent value of $\xi_{\mathrm{t}}$ can be recovered, so the constant microturbulence is adopted for the Sun, although its anisotropy and depth dependence are now well-established (Gray 1988).

\section{Results}

The relative $\mathrm{Mn}$ abundance ratios $[\mathrm{Mn} / \mathrm{Fe}]$ and their standard deviations $\sigma$ for all stars are listed in Table 1. Stars with metallicities higher than that of HD 102200 were analyzed directly relative to the Sun; in this case, the resonance triplet at $403 \mathrm{~nm}$ was not used for the reasons given above. The Mn abundances in stars more metal-poor than HD 102200 are calculated relative to this reference star, and corrected by an average $\mathrm{Mn}$ abundance derived for the HD 102200 relative to the Sun. These differential abundances are consistent within their $\sigma$ with $\mathrm{Mn}$ abundances in stars calculated from the excited lines strictly relative to the Sun.

Figure 6 shows $[\mathrm{Mn} / \mathrm{Fe}]$ ratios for all stars as a function of metallicity. The binaries are marked with open circles. The most interesting result is that the NLTE abundances of Mn in all stars are systematically higher than the LTE abundances; as expected, the difference $[\mathrm{Mn} / \mathrm{Fe}]_{\mathrm{NLTE}}-[\mathrm{Mn} / \mathrm{Fe}]_{\mathrm{LTE}}$ increases with decreasing metallicity.

In the most metal-poor stars $([\mathrm{Fe} / \mathrm{H}] \leq-2.5)$, we find especially large NLTE corrections for the resonance triplet at $403 \mathrm{~nm}$. It is therefore to be expected that LTE abundances of Mn in spectra of halo stars, where only the resonance near-UV lines can be detected, are significantly underestimated. The dependency of NLTE effects on (a) stellar parameteres $\left(T_{\text {eff }}, \log g,[\mathrm{Fe} / \mathrm{H}], \xi_{\mathrm{t}}\right)$ and (b) excitation potential of a line is obvious, and it is important to keep it in mind even when LTE analysis is performed.

The most uncertain parameter in all NLTE calculations is $S_{\mathrm{H}}$, the scaling factor to the cross-sections for inelastic $\mathrm{H}$ collisions, which are calculated with Drawin's (1968) formula. Fortunately, the $[\mathrm{Mn} / \mathrm{Fe}]$ ratios are not very sensitive to the choice of $S_{\mathrm{H}}$, as long as very large scaling factors $\left(S_{\mathrm{H}} \gg 1\right)$ are not used. The test calculations for four metal-poor stars show (Fig. 7) that the change in a scaling factor $S_{\mathrm{H}}=0 \ldots 1$ leads to a variation in the $[\mathrm{Mn} / \mathrm{Fe}]$ ratios $\left(\Delta[\mathrm{Mn} / \mathrm{Fe}]_{0 . .1}\right)$ in the range of $+0.05 \ldots-0.1$ dex. For stars with $[\mathrm{Fe} / \mathrm{H}]>-1$, we derive $\Delta[\mathrm{Mn} / \mathrm{Fe}]_{0 . \ldots 1} \sim+0.03 \ldots-0.05$. The error bars in Fig. 7 denote standard deviations $\sigma$ of $[\mathrm{Mn} / \mathrm{Fe}]$ values. Obviosuly, small $\sigma$ are achieved with $S_{\mathrm{H}} \leq 0.5$. It is reasonable to suggest that $S_{\mathrm{H}}=0.05$ (our reference value) provides the smallest scatter between different lines when all four stars are taken into account. This value

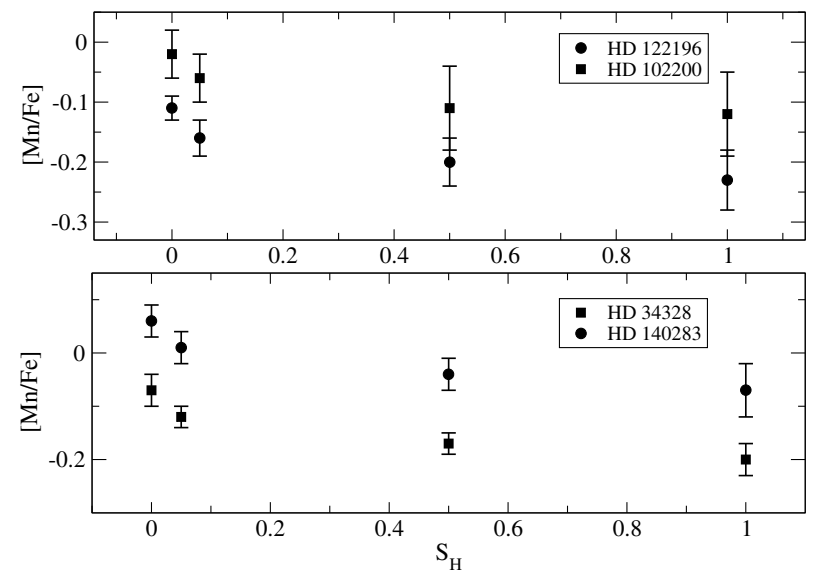

Fig. 7. $[\mathrm{Mn} / \mathrm{Fe}]$ ratios for HD 34328, HD 102200, HD 140283, and HD 122196 calculated with $S_{\mathrm{H}}=0,0.05,0.5$, and 1 .

agrees with the results derived for $\mathrm{Ba}$ and $\mathrm{Eu}$ by Mashonkina (1996) and for Mg emission lines by Sundquist et al. (2008), who present convincing arguments in favor of very low $(\ll 1)$ but non-zero values for $S_{\mathrm{H}}$. Also, according to Belyaev \& Barklem (2003), the standard Drawin's formula $\left(S_{\mathrm{H}}=1\right)$ strongly overestimates the rate of transitions for Li. If these estimates are true for $\mathrm{Mn}$ as well, we can claim only a slightly subsolar $[\mathrm{Mn} / \mathrm{Fe}]$ trend with decreasing metallicity. The variation in $S_{\mathrm{H}}$ within the range $0 \ldots 1$ will not change the general trend in Fig. 6 given the large differences $[\mathrm{Mn} / \mathrm{Fe}]_{\mathrm{NLTE}}-[\mathrm{Mn} / \mathrm{Fe}]_{\mathrm{LTE}}$ in metal-poor stars.

We also expect that a thermalizing effect of $\mathrm{H}$ collisions in metal-poor stars would be compensated by enhanced photoionization rates. In Papers I and II, we showed that a hydrogenic approximation for photoionization cross-sections, which is the only one available to us, underestimates the NLTE effects in $\mathrm{Mn}$ I. The magnitude of underestimation increases in metal-poor stars, where the radiation field is stronger than in the Sun. We performed test NLTE calculations for the star HD 34328 with photoionization cross-sections for all levels increased by a factor of 300. The effect turned out to be significant, because Mn abundances derived from the excited lines increased by 0.4 dex, on average. The abundances determined from the resonance lines at $403 \mathrm{~nm}$ only change by +0.05 dex. Strongly different NLTE corrections required for the resonance and excited lines point at the inadequacy of a simple hydrogenic photoionization approximation. Most likely, cross-sections for particular low-excitation levels of Mn I exhibit resonance structures, as found for Fe I (Bautista 1997).

\subsection{Comparison with recent work}

Recently, comparative manganese abundance analyses of metalpoor stars in different populations have appeared in the literature, and all of them refer to LTE. The average Mn abundances for stars with metallicities $-1 \ldots 0$ are in agreement between the compiled analyses as to slope and absolute values. We find a more or less smooth increase in $[\mathrm{Mn} / \mathrm{Fe}]$ with $[\mathrm{Fe} / \mathrm{H}]$ in the results of Prochaska \& McWilliam (2000), Nissen et al. (2000), Reddy et al. (2003), Reddy et al. (2006), and Feltzing et al. (2007). The Mn abundances in stars with lower metallicities, $[\mathrm{Fe} / \mathrm{H}]<-1$, show a relatively large scatter (Prochaska \& McWilliam 2000; Bai et al. 2004; Cayrel et al. 2004; Reddy et al. 2006; Lai et al. 2008). But it appears that the average [Mn/Fe] trend remains almost constant with the mean value -0.4 dex. 
Our NLTE Mn abundances are systematically larger than those from the comparison studies. The offset of +0.2 to +0.4 dex is mainly due to the NLTE effects, as can be predicted from Table 3. In contrast, the Mn underabundance suggested by our LTE analysis is consistent with the results of the others. The LTE values of Prochaska \& McWilliam (2000) corrected for mean NLTE effects from Table 3 agree reasonably well with our NLTE Mn abundances in the metal-poor stars $(-2<[\mathrm{Fe} / \mathrm{H}]<$ $-1)$ at a constant level of $[\mathrm{Mn} / \mathrm{Fe}] \sim-0.1 \ldots 0.0$. Also, applying NLTE corrections to the data of Lai et al. (2008), we can see that manganese follows the depletion of iron even in the most metalpoor stars. For stars with $-2.5<[\mathrm{Fe} / \mathrm{H}]<-1.5$, our results slightly deviate from the corrected LTE abundances presented by McWilliam et al. (2003). The reason for this discrepancy is that in this metallicity range McWilliam et al. (2003) used the LTE results of Johnson (2002), which were obtained by analyzing only giant stars. Here, we note that work on incoherent data compiled from various sources also tends to hide much of the chemical evolution of the Galaxy in an exceedingly large scatter.

Unfortunately, the results presented by Feltzing et al. (2007) are for disk stars alone, which are outside the range of metallicities covered by our stellar sample. However, their finding of different abundance ratios $[\mathrm{Mn} / \mathrm{O}]$ in stars of thick and thin disks is not surprising, because it reproduces a similar effect for the [Fe/Mg] ratio (Fuhrmann 2004).

\subsection{Mn nucleosynthesis and chemical evolution}

Manganese is a neutron-rich element in the sense that it has a greater neutron-proton excess than the adjacent elements. It could therefore be sensitive to the neutron excess available to the progenitor star in either the carbon-burning or silicon-burning phases. Following the bimodal enrichment scheme originally proposed by Arnett (1971; see also Woosley \& Weaver 1982), the yields of the neutron-rich elements always seem to depend on the metals available to the star when the metal abundance of the progenitor becomes larger than $[\mathrm{Fe} / \mathrm{H}] \sim-1.5($ or $[\alpha / \mathrm{H}]>$ -1.0 ), as long as the elements do not originate in a complete $\mathrm{Si}$ burning environment. Therefore, the stars with $[\mathrm{Fe} / \mathrm{H}]>-1.5$ must have been fed by $\mathrm{SNe}$ of a second or later generation.

Taking into account possible residual systematic errors due to underestimating photoionization by the hydrogen-like approximation, we can claim that $[\mathrm{Mn} / \mathrm{Fe}]$ is only slightly subsolar or even solar throughout the range of metal abundances analyzed here. This would be confirmed by the results of Feltzing et al. (2007) for the disk stars after applying the necessary NLTE corrections, whereas it does not fit the earlier work of Gratton (1989) very well. The models of chemical evolution presented by Timmes et al. (1995) are, however, fully inadequate for representing the $[\mathrm{Mn} / \mathrm{Fe}]$ ratio in metal-poor stars. Based on the discussion in Umeda \& Nomoto (2002), it is tempting to ascribe the discrepancy to the choice of the mass cut. Whether the required increase in the mass cut or a change in the neutron excess is still compatible with the observed Co abundances, will be examined in a future paper.

Acknowledgements. M.B. acknowledges with gratitude the Max-Planck Institute for Extraterrestrial Physics (Germany) for her PhD fellowship.

\section{References}

Abt, A. 1952, ApJ, 115, 199

Anstee, S. D., \& O'Mara, B. J. 1995, MNRAS, 276, 859

Arnett, W. D. 1971, ApJ, 166, 153

Asplund, M., Grevesse, N., Sauval, A. J., et al. 2005a, A\&A, 435, 339

Asplund, M., Grevesse, N., Sauval, A. J., et al. 2005b, A\&A, 431, 693

Bagnulo, S., Jehin, E., Ledoux, C., et al. 2003, ESO Messenger, 114, 10

Bai, G. S., Zhao, G., Chen, Y. Q., et al. 2004, A\&A, 425, 671

Baumüller, D., \& Gehren, T. 1997, A\&A, 325, 1088

Bautista, M. A. 1997, A\&AS, 122, 167

Belyaev, A. K., \& Barklem, P. S. 2003, Phys. Rev. A, 68, 062703

Bensby, T., Feltzing, S., Lundström, I., et al. 2005, A\&A, 433, 185

Bergemann, M., \& Gehren, T. 2007, A\&A, 473, 291 (Paper I)

Blackwell-Whitehead, R. J., Pickering, J. C., Pearse, O. et al. 2005, ApJS, 157, 402

Blackwell-Whitehead, R. J., \& Bergemann, M. 2007, A\&A, 472, L43 (Paper II)

Booth, A. J., Blackwell, D. E., Petford, A. D., et al. 1984, MNRAS, 208, 147

Bruls, J. H. M. J. 1993, A\&A, 269, 509

Butler, K., \& Giddings, J. 1985, Newsletter on the Analysis of Astronomical Spectra No. 9, University of London

Cayrel, R., Depagne, E., Spite, M., et al. 2004, A\&A, vol. 416, 1117

del Peloso, E. F., Cunha, K., da Silva, L., et al. 2005, A\&A, 441, 1149

Drawin H.-W. 1968, Z. Phys., 211, 404

Feltzing, S., Fohlmann, M., \& Bensby, T. 2007, A\&A, 467, 665

Fuhrmann K., 2004, Astron. Nachr., 325, 3

Fuhrmann, K., Axer, M., \& Gehren, T. 1993, A\&A, 271, 451

Fuhrmann, K., Pfeiffer, M., Frank, C., et al. 1997, A\&A, 323, 909

Gehren, T., Liang, Y. C., Shi, J. R., Zhang, H. W., \& Zhao, G., 2004, A\&A, 413, 1045

Gehren T., Shi, J. R., Zhang, H. W., et al. 2006, A\&A, 451, 1065

Gratton, R. G. 1987, ESO Conf. Workshop Proc., 27, 153

Gratton, R. G. 1989, A\&A, 208, 171

Gray, D. 1988, Lectures on spectral-line analysis of F, G, and K stars, The publisher: Box 141, Arva, Ontario NOM 1C0, Canada

Grupp, F. 2004, A\&A, 420, 289

Heger, A., \& Woosley, S. E. 2008, ApJ, submitted [arXiv: 0803. 3161]

Helfer, H. L., Wallerstein, G., \& Greenstein, J. L. 1959, ApJ, 129, 700

Johnson, J. A. 2002, ApJS, 139, 219

Korn, A., Shi, J. R., \& Gehren, T. 2003, A\&A, 407, 691

Kurucz, R. L. 1992, Rev. Mex. Astron. Astrofis., 23, 45

Lai, D., Bolte, M., Johnson, J., et al. 2008, ApJ, 681, 1524

Lodders, K. 2003, ApJ, 591, 1220

Mashonkina, L. 1996, in Model Atmospheres and Spectrum Synthesis, ed. S. J. Adelman, F. Kupka, \& W. W. Weiss, 108, ASP Conf. Ser., 140

Mashonkina, L., Gehren, T., Travaglio, C., \& Borkova, T., 2003, A\&A, 397, 275

Mashonkina, L., Korn, A. J., \& Przybilla, N. 2007, A\&A, 461, 261

Mashonkina, L., Zhao, G., Gehren, T., et al. 2008, A\&A, 478, 529

McWilliam, A., Rich, R. M., \& Smecker-Hane, T. A. 2003, ApJ, 592, L21

Nissen, P. E., Chen, Y. Q., Schuster, W. J., et al. 2000, A\&A, 353, 722

Prochaska, J. X., \& McWilliam, A. 2000, ApJ, 537, L57

Reddy, B. E., Tomkin, J., Lambert, D. L., \& Allende Prieto, C. 2003, MNRAS, 340,304

Reddy, B. E., Lambert, D. L., \& Allende Prieto, C. 2006, MNRAS, 367, 1329

Ryan, S. G., Norris, J. E., \& Beers, T. C. 1996, ApJ, 471, 254

Sobeck, J. S., Simmerer, J. A., Fulbright, J. P. et al. 2005, 336, ASP Conf. Ser., 363

Sobeck, J. S., Ivans, I. I., Simmerer, J. A., et al. 2006, ApJ, 131, 2949

Steenbock, W., \& Holweger, H. 1984, A\&A, 130, 319

Sundqvist, J. O., Ryde, N., Harper, et al. 2008, A\&A, 486, 985

Takeda, Y., \& Honda, S. 2005, PASJ, 57, 65

Timmes, F. X., Woosley, S. E., \& Weaver, T. A. 1995, ApJS, 98, 617

Umeda, H., \& Nomoto, K. 2002, ApJ, 565, 385

VandenBerg, D. A., Swenson, F. J., Rogers, F. J., et al. 2000, ApJ, 532, 430

Wheeler, J. C., Sneden, C., \& Truran, J. W. 1989, ARA\&A, 27, 279

Woosley, S. E., \& Weaver, T. A. 1982, in Essays on Nuclear Astrophysics, ed.

C.A. Barnes, D.D. Clayton, \& D.N. Schramm (Cambridge Univ. Press), 377 